Cahiers de philosophie de l'université de

Libres résonances entre sculpture et phénoménologie : Martin Heidegger et Anthony Caro

Hadrien France-Lanord

\title{
OpenEdition
}

Journals

Édition électronique

URL : https://journals.openedition.org/cpuc/297

DOI : $10.4000 /$ cpuc. 297

ISSN : 2677-6529

Éditeur

Presses universitaires de Caen

Édition imprimée

Date de publication : 1 novembre 2018

Pagination : 95-116

ISBN : 978-2-84133-904-4

ISSN : $1282-6545$

Référence électronique

Hadrien France-Lanord, « Libres résonances entre sculpture et phénoménologie : Martin Heidegger et Anthony Caro », Cahiers de philosophie de l'université de Caen [En ligne], 55 | 2018, mis en ligne le 01 novembre 2019, consulté le 01 février 2023. URL : http://journals.openedition.org/cpuc/297 ; DOI : https://doi.org/10.4000/cpuc.297

\section{(ब) $(1) \Theta$}

Creative Commons - Attribution - Pas d'Utilisation Commerciale 4.0 International - CC BY-NC 4.0 https://creativecommons.org/licenses/by-nc/4.0/ 


\title{
Libres résonances entre sculpture et phénoménologie: Martin Heidegger et Anthony Caro
}

\author{
Verkörperung und Kunstwerk ${ }^{1}$ \\ Prendre corps et œuvre d'art \\ Martin Heidegger
}

\begin{abstract}
Darmi les derniers textes que Heidegger a consacrés à la question de l'œuvre d'art se trouvent deux minces plaquettes écrites l'une et l'autre à l'occasion de la rencontre qu'il a faite de l'œuvre sculptée de deux de ses contemporains. Le premier texte, Remarques sur art-sculpture - espace, a été prononcé le 3 octobre 1964 à l'occasion de l'inauguration de l'exposition d'œuvres de Bernhard Heiliger à la galerie Im Erker à Saint-Gall. L'autre texte, L'art et l'espace, a été écrit en dialogue avec l'œuvre d'Edouardo Chillida dont Heidegger a fait la connaissance également à la galerie Im Erker en 1968. Heinrich Wiegand Petzet n'était pas présent lors de cette première rencontre, mais c'est lui qui en fut d'une certaine manière l'initiateur, parce que c'est lui qui le premier a parlé à Heidegger du travail de Chillida au début des années 1960, ainsi qu'il le raconte lui-même:
\end{abstract}

1. C'est une notation isolée de Heidegger, qui se trouve dans les notes préparatoires en vue du séminaire qui s'est tenu entre le 18 et le 21 mars 1969 à Zollikon à l'invitation de Medard Boss. Elle est consignée dans le volume 89 de l'édition intégrale, récemment paru (Zollikoner Seminare, GA 89, 629). On pourrait aussi traduire Verkörperung par «incarnation », si le mot ne se prêtait pas à un malentendu que Heidegger cherche à dissiper dans une autre note où il emploie le verbe sich inkarnieren: "La spatialité du Dasein se développe dans son être-en-corps [Leiblichkeit]. Que veut dire ce "développe"?/ Cela ne veut dire ni que le Dasein se localise, $n i$ qu'il s'incarne par son corps - cela ne veut pas dire non plus que la spatialité serait d'abord constituée par l'être-en-corps» (GA 89, 425). L'expression "prendre corps", qui est une manière de dire en français le déploiement de quelque chose, cherche à éviter la dimension trop "physique» que peut avoir le mot incarnation, alors qu'il s'agit de questions phénoménologiques et de déploiement d’une phénoménalité. 
C'est ainsi qu'à partir de surfaces sans soudures ni ruptures naissait une gestuelle formelle très austère, où rien n'était laissé au hasard. Le sculpteur avait dit: «Ce n'est pas la forme qui m'importe, c'est le rapport des formes les unes avec les autres - la relation qu'il y a entre elles. » Et l'on touche ici à ce qui constitue pour ce sculpteur le problème fondamental: l'inclusion «de l'espace» dans son travail.

Heidegger avait dressé l'oreille. Depuis sa conférence de Darmstadt Bâtir habiter penser, la question de l'espace n'avait cessé de l'occuper - la question de savoir si l' «espace» de Galilée et de Newton, l'espace de la science moderne de la nature, était identique à l' « espace» de l'art. Comment Chillida entendait-il donc $l^{\prime}$ «espace $»^{2}$ ?

Comme témoignage de l'importance réciproque que cette rencontre a eu pour le sculpteur et pour le penseur, il existe une édition de L'art et l'espace écrite de la main de Heidegger avec des œuvres originales de Chillida. Sur une suggestion de Franz Larese, Heidegger a également inscrit son texte à la main sur des pierres lithographiques exposées en ce moment (5 décembre 2017-15 avril 2018) au musée Guggenheim de Bilbao dans le cadre de l'exposition "L'art et l'espace » réalisée à l'initiative de Manuel Cirauqui. L'un et l'autre partageaient l'expérience pensante de travailler avec leurs mains et l'idée qu'un même espace de pensée pouvait prendre corps à travers des mots ou à travers des œuvres sculptées. Dans le bureau de la maison de Heidegger à Zähringen était accrochée au mur une petite eau-forte de mains, que Chillida avait offerte au penseur.

Ces deux courts textes, Remarques sur art - sculpture - espace et L'art et l'espace, sont essentiels et très précieux, parce qu'on y trouve la formulation ultime de la pensée heideggerienne de l'espace. Marlène Zarader conclut à juste titre son texte sur «Le lieu de l'art» en soulignant que:

[...] c'est à l'occasion de sa réflexion sur l'art que Heidegger fut conduit à redonner son unité au monde et sa densité à la chose, et c'est en prenant appui sur la chose ainsi comprise qu'il put penser autrement l'espace (autrement, c'est-à-dire non plus à partir du Dasein, mais à partir du lieu dessiné par les choses elles-mêmes). Ressaisi dans cette perspective, L'art et l'espace constitue moins un prolongement de la réflexion antérieure qu'une confirmation de celle-ci. Si, comme nous le croyons, c'est bien la pensée de l'art qui permit la redéfinition du lieu, alors la réflexion tardive qui s'efforçait de revenir du lieu à l'art ne pouvait prendre d'autre allure que celle d'une reconnaissance, d'autre forme que celle d'une récapitulation de son propre parcours ${ }^{3}$.

2. H. W. Petzet, Le chemin de l'étoile. Rencontres et causeries avec Heidegger, 1929-1976, C.-N. Grimbert, P. Arjakovsky (trad.), Paris, Éditions du Grand Est, 2014, p. 180.

3. M. Zarader, «Le lieu de l'art», Les Temps Modernes, $\mathrm{n}^{\circ}$ 650, juillet-octobre 2008, Heidegger. Qu'appelle-t-on le Lieu?, p. 116. Dans le livre qui est à ma connaissance le plus complet sur les rapports entre Heidegger et la sculpture, Andrew J. Mitchell conclut ainsi son 
Il est significatif de la pratique phénoménologique de Heidegger que ce soit à partir de l'expérience qu'il a pu faire en corps de sculptures contemporaines (pour l'essentiel abstraites) qu'il est parvenu aux derniers développements de sa pensée de l'espace et du corps. Exactement à la même période, c'est aussi dans l'expérience elle-même incarnée du dialogue avec Medard Boss et un cercle de psychiatres qu'il approfondit ces questions de la spatialité, de l'être-en-corps et de leurs rapports. C'est un des enseignements majeurs de Être et temps: il n'y a pas de phénoménologie sans être au monde.

\section{Le déploiement de l'espace et le phénomène du corps}

La pensée heideggerienne de l'espace a connu plusieurs évolutions et remaniements notoires dont Didier Franck, notamment, a retracé une partie dans Heidegger et le problème de l'espace, ainsi que dans le texte "Le séjour du corps» qui accompagne sa traduction des Remarques sur art - sculpture - espace et dans lequel il expose comment la première approche de la question dans Etre et temps a été entièrement reprise dès lors que l'espace «doit être pensé depuis l'Ereignis en tant que monde où l'être s'évanouit ${ }^{4}$. En effet, la publication des Apports à la philosophie aura apporté de nouveaux éléments et de nouvelles perspectives que Heidegger lui-même ne cesse de reprendre au moins jusqu'aux deux plaquettes évoquées 5 . Quant à la question du corps, qui n'est pratiquement pas développée comme telle dans Être et temps car Heidegger a conscience qu'elle "abrite une problématique spécifique» (\$23), c'est aussi grâce aux développements de la pensée de l'histoire de l'être à partir des années 1930, qu'elle parviendra à se formuler pleinement. On trouve dans le cours sur Nietzsche du semestre d'été 1939 un des premiers échos les plus développés de cette pensée dont l'horizon ultime sera de tenter de dire «die welthafte

introduction: «Heidegger's engagements with sculpture in the 1960 are thus deeply enmeshed in his earlier thinking. They emerge from a rethinking of body and space that departs from the earlier conceptions of Being and Time and continues the trajectory of inquiry opened in "The Origin of the Work of Art", developing these latter ideas in a more explicitly corporeal vein than ever before in his work. Heidegger's texts present us with a thought of the mutual belonging together of space and body, a thought that allows the art of sculpture to touch us so " (Heidegger among the Sculptors. Body, Space and the Art of Dwelling, Stanford, Stanford University Press, 2010, p. 14).

4. D. Franck, «Le séjour du corps», Les Temps Modernes, $\mathrm{n}^{\circ}$ 650, p. 62.

5. La première, Remarques sur art - sculpture - espace, a été traduite en 2008 par Didier Franck dans Les Temps Modernes ( $\mathrm{n}^{\circ}$ 650), accompagnée d'un texte dans lequel Didier Franck reprend et poursuit, au vu des éléments nouveaux, son propre travail. La seconde, L'art et l'espace, a paru en bilingue du vivant de Heidegger en 1969 dans une plaquette chez Erker-Verlag, avec une traduction due à Jean Beaufret et François Fédier (reprise dans Questions IV, Paris, Gallimard, 1976). 
Leiberfahrung», pour reprendre une note de $1965^{6}$, c'est-à-dire : comment l'expérience du corps fait monde, comment l'épreuve humaine d'être en corps se déploie comme monde.

Dans le cours sur Nietzsche, Heidegger travaille sur deux fronts distincts qui ne se situent pas sur le même plan. Mais en amont de ces deux fronts, il s'agit d'abord de sortir de l'horizon métaphysique de la distinction du corps et de l'âme. Voici le passage principal:

La tonalité [Stimmung] qui nous dispose est précisément la manière fondamentale selon laquelle nous sommes au dehors de nous-mêmes. Mais c'est ainsi essentiellement que nous déployons notre être et que nous sommes constamment.

Dans tout cela, l'état du corps entre en vibration, état qui nous élève loin par-delà nous-mêmes ou qui laisse l'homme s'empêtrer en lui-même dans un abrutissement. Nous ne sommes pas d'abord «vivant», état auquel il faudrait en outre ajouter tout un appareillage appelé corps; au contraire, nous ne vivons que pour autant que nous donnons corps [wir leben, indem wir leiben]. Cette manière de donner corps [dieses Leiben] est dans tout son déploiement quelque chose d'essentiellement autre que d'être seulement flanqué d'un organisme. Pour l'essentiel, ce que nous savons des sciences naturelles relativement au corps et à sa manière de se déployer en donnant corps, ce sont des constatations objectives, dans lesquelles le corps est d'avance mésinterprété comme corps purement physique [Körper $]^{7}$.

Le premier front par rapport auquel se situe Heidegger dans son articulation entre corps et espace est la pensée de Husserl qui « continue, écrit Françoise Dastur, de penser l'être humain en accord avec toute la tradition métaphysique comme union du sensible et de l'intelligible» ${ }^{8}$. La notion de Stimmung, de tonalité, qui est, au même titre que l'entente [das Verstehen], un mode de dévoilement de ce qui est, permet en revanche à Heidegger de quitter dès Être et temps cette conception du corps et de l'esprit. Il ne faut pas négliger «die Tragweite des Eröffnens der Stimmung» comme dira Heidegger ${ }^{9}$, c'est-à-dire la portée du mouvement d'ouverture qu'est la tonalité. Cette dimension primordiale d'ouverture tonale de l'être humain au monde fait que le Dasein est toujours d'emblée au-dehors, notamment hors de la sphère de la subjectivité transcendantale husserlienne, qui reste théorique et close, malgré la structure intentionnelle de la conscience.

6. M. Heidegger, GA 89, 447.

7. M. Heidegger, GA 6.1, 100; trad. fr.: Nietzsche I, P. Klossowski (trad.), Paris, Gallimard (Bibliothèque de philosophie), 1990, p. 96.

8. F. Dastur, Husserl. Des mathématiques à l'histoire, Paris, PUF, 1999, p. 106.

9. M. Heidegger, Zu eigenen Veröffentlichen, GA 82, 76 . 
Cet être-au-dehors est aussi une mise au diapason (toute Stimmung est un stimmen), qui décentre l'homme pour en faire chaque fois l'élément d'une relation à une altérité: altérité des autres, altérité du soi, altérité du monde - et en premier lieu: altérité de l'être même. Dans cette possibilité d'accord - et de désaccord - avec les autres, avec soi ou le monde, la notion de Stimmung rappelle que le corps est toujours impliqué, aussi bien dans des phénomènes comme le rougissement, le mal de dos ou le deuil, ainsi que le montre Heidegger, mais aussi dans l'écoute et la parole et dans tout l'être-au-monde. Le corps dont parle Heidegger dans le cours sur Nietzsche et dont il développe les modalités d'ouverture, de mise en vibration tonale, ainsi que les gestes dans le séminaire de 1965 avec Medard Boss, est un corps qui n'est pas simplement $i c i$, au sens géolocalisable du terme ${ }^{10}$, ni un corps dont les limites coïncident avec la limite physique de la peau ${ }^{11}$. C'est un corps qui est à penser à partir de l'ouverture de l'être-au-monde. Ainsi, dès Etre et temps, la pensée de la tonalité comprend en puissance ${ }^{12}$ une pensée du corps qui passe outre la conception métaphysique de l'homme comme union de l'âme et du corps, qui se libère de la centralité forclose du pôle subjectif husserlien, et qui renverse du même coup l'articulation husserlienne entre le corps et l'espace. En effet, Husserl pense l'espace à partir du corps compris comme centre perceptif, ainsi que l'explique Dominique Pradelle dans son texte «Comment penser le propre de l'espace?» qui commence par un exposé de «La constitution husserlienne de la spatialité » pour montrer comment Heidegger s'en démarque:

10. Cf. M. Heidegger, GA 89, 780 ; trad. fr.: Séminaires de Zurich, M. Boss (éd.), C. Gros (trad.), Paris, Gallimard, 2010, p. 137: «Mon corps n'est pas l'ici. Où est mon corps? Comment déterminez-vous l'ici? Où suis-je, où êtes-vous? Quelle est la question importante et difficile dont il s'agit ici ? Il s'agit manifestement de la question : comment le corps se rapporte à l'espace? Manifestement le corps se rapporte à l'espace d'une façon totalement différente de la façon dont, par exemple, une chaise est là (à notre disposition) dans l'espace. Le corps occupe un espace en y prenant place. Est-il délimité par l'espace? Où s'étendent les limites du corps? Où le corps finit-il?» - Réponse de Bergson dans Les deux sources de la morale et de la religion: "Car si notre corps est la matière à laquelle notre conscience s'applique, il est coextensif à notre conscience, il comprend tout ce que nous percevons, il va jusqu'aux étoiles».

11. Cf. M. Heidegger, GA 89, 782-783; Séminaires de Zurich, p. 140 : «La limite de mon corps physique [Körper] ne devient jamais elle-même - bien qu'en apparence elle recouvre la limite du corps $[$ Leib $]$ - une limite du corps».

12. Dans les notes préparatoires pour le séminaire de 1965 avec Boss (GA 89, 452-456), on trouve un ensemble intitulé «Leiblichkeit/Befindlichkeit» que Heidegger renvoie au $\$ 28$ d'Être et temps, qui est le paragraphe où, juste avant d'amener les deux modalités primordiales d'êtreouvert que sont la tonalité et l'entente, Heidegger rompt avec la pensée de la conscience en développant la signification du $D a$ de Dasein, non pas au sens spatial, mais en tant que dimension primordiale de décloisonnement et d'apérité. C'est en quelque sorte l'adieu à la conscience. 
Le corps charnel possède, dans cette progression des champs sensoriels vers l'espace, une fonction centrale: ici absolu, point-zéro de l'orientation, support des champs sensoriels et structure unitaire des systèmes kinesthésiques (c'està-dire des degrés de liberté de mes mouvements), il permet l'élargissement progressif des champs sensoriels bidimensionnels à l'espace tridimensionnel grâce à la mise en jeu de nouveaux degrés kinesthésiques - mouvements des yeux, de la tête, du tronc et finalement de la marche, qui permet la constitution d'un horizon spatial indéfini, homogène et isotrope, système de lieux que je puis idéalement atteindre en m'y rendant ${ }^{13}$.

Dans des notes préparatoires au séminaire de 1965 avec Boss, Heidegger consigne ce qui le distingue ici de Husserl:

Là où mon corps est présent - c'est là que je ne suis pas - | parce que mon «être» n'est pas simplement présent là-devant -

Là où mon corps se déploie en corps - se comporte par des gestes - dans une contrée (au-dessus - en-dessous / devant - derrière) - là-bas - là je n'y suis pas - en tant qu' «ego»-Sujet| pôle du moi - mais au contraire: en séjournant je suis auprès de et ensemble ${ }^{14}$.

S'agissant de l'articulation entre le corps et l'espace dans la phénoménologie de Heidegger, on peut résumer le renversement par rapport à Husserl avec cette formulation de 1965: «Le Dasein n'est pas spatial parce qu'il est corporel, au contraire: la corporéité n'est possible que parce que le Dasein est spatial au sens où il fait espace $»^{15}$. Or faire espace [einräumen], déployer l'espace [räumlichen] ou donner lieu [örtern], cela se comprend chez Heidegger à partir de l'être-au-monde et de la déclosion de l'ouvert en lequel ce qui est peut se déployer en son espace propre. Ce n'est pas pour Heidegger une question de perception, mais de déploiement d'être. Prenons un exemple: quand on regarde L'atelier rouge de Matisse, nos yeux perçoivent bel et bien du pigment rouge; mais, cette perception sensible de pigment ne nous met pas pour autant en rapport avec la manière dont le tableau fait picturalement espace (ce qui ne peut en outre se produire que si notre corps est présent au tableau, et non pas grâce à la perception visuelle d'une reproduction, si bonne soit-elle). L'espace du tableau, que Matisse appelle tantôt espace pictural, tantôt espace spirituel ou espace plastique, ne se laisse pas rencontrer à l'aune de la perception; c'est un espace ouvert et

13. D. Pradelle, "Comment penser le propre de l'espace?", Les Temps Modernes, $\mathrm{n}^{\circ}$ 650, p. $80-81$.

14. M. Heidegger, GA 89, 412 .

15. M. Heidegger, GA 89, 775; Séminaires de Zurich, p. 132. Voir aussi GA 89, 629: «Le Da-sein ne devient pas spatial du fait qu'il est corporel, au contraire, il ne peut être en corps que dans la mesure où il déploie l'espace à sa manière [räumlicht] | donne lieu [Örtert]». 
déclos ( «un espace cosmique dans lequel on ne sentait pas plus les murs, que le poisson dans la mer ${ }^{16}$, dit le peintre), un espace de phénoménalité dans lequel le "phénomène du corps [Leibphänomen] », comme dit Heidegger, peut se mouvoir, c'est-à-dire exister et habiter. On peut séjourner dans l'espace pictural de L'atelier rouge, à la condition de le laisser se déployer, alors que la perception nous livre une objectivation qui met à distance. De même, à travers l'épreuve vibrante de la tonalité, comme y insiste Heidegger à propos du rougissement du visage, le phénomène du corps nous met toujours d'emblée en rapport avec autrui et la spatialité propre d'une personne ${ }^{17}$ (qu'elle soit ou non dans notre champ de perception).

Du point de vue phénoménologique de Heidegger, l'essentiel est l'attention à la phénoménalité de la chose à partir d'elle-même; un phénomène, écrit-il, est «ce qui se montre, quand je pense et que j'essaie de regarder quelque chose en tant que tel - dans ce qu'il a de propre» ${ }^{18}$. Il faut donc essayer de faire une expérience de l'espace en ce qu'il a de propre, sans le reconduire à quelque chose d'autre que lui comme le fait la représentation courante:

Car la représentation commune tient quelque chose pour éclairci seulement quand il est expliqué, c'est-à-dire reconduit à quelque chose d'autre, et dans le cas présent: l'espace en tant qu'il est rapporté au corps physique ${ }^{19}$.

C'est le même verbe, "reconduire à [zurückführen]», que Heidegger emploie au début de la séance du 14 mai 1965 en évoquant les phénomènes du corps $^{20}$. Dans l'extrait du cours sur Nietzsche de 1939, il souligne que le corps ne doit pas être reconduit à l'idée d'organisme et à la conception biologique de la vie dont elle émane. Ainsi apparaît le deuxième front par rapport auquel se situe le propos: Heidegger parle ici en 1939, alors que, sur un fond de

16. H. Matisse, Écrits et propos sur l'art, D. Fourcade (éd.), Paris, Hermann, 1992, p. 104

17. M. Heidegger, GA 89, 788; Séminaires de Zurich, p. 145: «D’ordinaire nous prenons le rougissement en tant qu'expression, c'est-à-dire que nous le tenons d'emblée pour signe d'un état psychique intérieur. Mais qu'y a-t-il dans le phénomène du rougissement lui-même? C’est aussi un geste, pour autant que celui qui rougit est relié aux êtres humains qui partagent sa vie. Vous voyez par là comment la corporéité a ce sens ekstatique caractéristique. Je souligne cela avec tant de netteté afin de vous éloigner de l'interprétation fallacieuse. Même les psychologues français interprètent tout de travers en tant qu'expression de quelque chose d'intérieur, au lieu de considérer le phénomène du corps dans sa liaison aux autres êtres humains».

18. M. Heidegger, GA 89, 385 .

19. M. Heidegger, Bemerkungen zu Kunst - Plastik - Raum, Saint-Gall, Erker-Verlag, 1996, p. 12; trad. fr.: Remarques sur art - sculpture - espace, D. Franck (trad.), Les Temps Modernes, $\mathrm{n}^{\circ} 650$, p. 51 .

20. Cf. M. Heidegger, GA 89, 781; Séminaires de Zurich, p. 138 : «il importe que nous laissions de prime abord ces phénomènes [corporels] être tels que nous les portons au regard sans chercher à les reconduire à quelque chose. En d'autres termes, il s'agit de faire abstraction de tous types de possibilités de re(con)duction». 
biologisme généralisé dans le monde occidental depuis la fin du XIX ${ }^{\mathrm{e}}$ siècle, triomphe en Allemagne la vision du monde raciste nazie qui reconduit tout ce qui est humain à une souche biologique et qui instaure totalitairement une nouvelle légalité, la prétendue "Lebensgesetzlichkeit» des historiens et des juristes nazis, c'est-à-dire la vie biologique du corps organique établie comme norme de tout ${ }^{21}$. Heidegger profite de ces développements pour soustraire la pensée de Nietzsche à la récupération nazie: «Il y a lieu d'insister une fois de plus qu'une caractérisation explicite ou implicite de cette métaphysique en tant que biologisme revient à ne rien penser du tout, et que toutes les références aux notions darwinistes doivent être écartées ${ }^{22}$, dit-il fermement plus loin dans le cours où il explique le sens de la pensée nietzschéenne du «corps comme fil conducteur». Dans Être et temps, Heidegger a souligné à deux reprises ( $\$ 9$ et 10) que la biologie ne peut être d'aucun secours en matière phénoménologique. Dans le cours sur Nietzsche, il coupe court à la reconduction hitlérienne fautive du corps humain à un pur organisme biologiquement vivant; il renverse même les choses en rapportant la vie - entendue en un sens pas strictement biologique - au déploiement du corps dans son acception phénoménologique et existentiale: "nous ne vivons que pour autant que nous donnons corps [wir leben, indem wir leiben] $\gg^{23}$. Heidegger travaille ainsi en sens inverse la résonance entre les deux mots étymologiquement apparentés que sont Leben [vie, vivre] et Leib [corps], de sorte que la vie proprement humaine prend sa consistance à partir de la manière dont nous donnons corps au monde et à notre propre corps. C'est le verbe leiben ${ }^{24}$ qui déplace les choses; je tente ici de le traduire par "donner corps», expression qui confère au mot " corps» une belle amplitude phénoménologique, parce qu'elle dit comment quelque chose a lieu et se déploie, mais on pourrait aussi le rendre par «déployer en corps». Dans une note des Remarques sur art - sculpture - espace, Heidegger en précise ainsi le sens: «séjour dans le monde». Il faut souligner que c'est un verbe: en tant que phénoménologue, Heidegger s'intéresse moins à un étant ou à

21. Sur cette nouvelle «bionomie» nazie et le biologisme généralisé de la vision du monde nazie, voir le livre de J. Chapoutot, La loi du sang. Penser et agir en nazi, Paris, Gallimard, 2014, notamment p. 55.

22. M. Heidegger, GA 6.1, 510; Nietzsche I, p. 440.

23. M. Heidegger, GA 6.1, 100; Nietzsche I, p. 96.

24. Sur ce verbe et le sens que lui confère Heidegger, voir ces lignes d'Ingrid Auriol dans son ouvrage Intelligence du corps, Paris, Cerf, 2013, p. 216: "Ce n'est pas un néologisme mais un ancien mot dont la langue d'aujourd'hui garde mémoire dans l'expression: “wie er leibt und lebt", que l'on emploie couramment pour signifier "la manière de vivre typique de quelqu'un" sans que, pour autant, le verbe leiben y soit entendu à la lettre. Avec Leiben, Heidegger indique que le Dasein "existe" le corps qui est à chaque fois le sien, sa manière d'être-corps détermine son séjour». 
une essence qu'à un mouvement et à une modalité d'apparaître; sa question est de savoir comment a lieu le corps, comment il se déploie et apparaît, et comment l'apparaître de ce déploiement humain en corps est à son tour une dimension de phénoménalité pour les autres et pour le monde. Rien de statique ici: comme l'existence, le corps humain est factif; nous avons à l'exister et à tout faire pour lui donner l'ampleur de dévoilement à la fois tonal et ententif dont il est capable. Le corps phénoménologique que pense Heidegger n'est pas moins vibratoire que pensant, il est existentialement une possibilité parmi les plus éminentes pour le monde de se déployer comme phénomène, à partir de l'expérience de la déclosion spatiale de l'existence humaine qui n'est pas au monde sans aménager les choses, les événements ou les rencontres en autant d'espaces où quelque chose peut avoir lieu.

\section{Le phénomène du corps et l'art moderne}

Ce rôle essentiel du déploiement tonal et pensant du corps, cette implication nouvelle du phénomène du corps dans la phénoménalité du monde, qui trouve à se formuler grâce à la phénoménologie (non seulement celle de Husserl et de Heidegger, mais aussi Merleau-Ponty, Levinas, Sartre, Henry et d'autres encore) - bref, cette révolution phénoménologique du corps en philosophie me semble tout à fait analogue à bien des égards à la révolution du corps qui s'est produite quelques décennies auparavant grâce à la naissance de l'art moderne. Ce n'est pas un hasard si c'est en dialogue avec deux sculpteurs modernes que Heidegger parvient à ses formulations ultimes sur le corps et l'espace, d'autant plus qu'il signale dans une note préparatoire, parmi les «relations insignes à l'espace», le rapport «artistique: architecture - sculpture - peinture ${ }^{25}$. Que ce soit en architecture (Heidegger fit à Ronchamp, dans l'église de Le Corbusier, une «halte prolongée» dit Petzet), en sculpture ou en peinture, tout l'art moderne y compris en cinéma, en poésie et au premier chef en danse, est à la fois l'écho et l'exploration d'une corporéité nouvelle. «Je crois que pour être bien l'homme, la nature se pensant, écrit Mallarmé le 27 mai 1867 à Eugène Lefébure, il faut penser de tout son corps - ce qui donne une pensée pleine et à l'unisson comme ces cordes du violon vibrant immédiatement avec sa boîte de bois creux». En peinture, cette habitation nouvelle du corps, aussi bien chez Cézanne que Monet, Kandinsky ou Pollock, est liée au déploiement autonome de la couleur et d'un espace pictural qui n'est plus inféodé à la mise en scène d'une illusion tridimensionnelle. Comme l'écrit Clement Greenberg:

25. M. Heidegger, GA 89, 622. 
Le tableau est désormais devenu un objet qui relève littéralement du même ordre spatial que celui de notre corps, il n'est plus le véhicule d'un équivalent imaginé de cet ordre. Il a perdu son «intérieur» et devient presque tout «extérieur», entièrement surface plane. Le spectateur ne peut plus aller s'y réfugier pour fuir l'espace dans lequel lui-même se tient $[\ldots]^{26}$.

De manière générale, la spatialité révolutionnaire de l'art moderne est inconcevable sans un rapport au corps qui ne soit pas simplement physico-sensible, mais: mondial. Le corps humain n'est pas une machine physique et animale, mais une possibilité de donner au monde son relief et son sens. Qu'on songe au Troisième quatuor à cordes de Giacinto Scelsi, à Vir Heroicus Sublimis de Barnett Newman, ou à la composition par champ que prône Charles Olson dans les belles pages sur «Le vers projectif». Dans les années 1950, Olson fut à la tête du Black Mountain College où se trouvait également Merce Cunningham, avec qui, de manière irréversible, la danse ne suit plus une mélodie extérieure à celle du corps lui-même, de sorte que le corps se met à danser en tant que corps, dans un espace ouvert, sans centre, où «tout endroit de la scène est également intéressant» dit Cunningham, qui demande également à ses danseurs «que les mouvements soient exécutés clairement, sans y faire entrer aucune expressivité. Ce doit être vraiment le mouvement qu'on voit en train de se faire chez tel ou tel danseur, et pas quelque chose qu'il ou elle ajoute et qui rend la vision plus difficile. Je veux voir la forme que prend tel mouvement sur tel danseur et rien d'autre ${ }^{27}$. Le corps n'étant plus physique, par opposition à intellectuel, plus exactement le corps n'étant plus pris dans l'opposition du sensible et de l'intelligible, il peut devenir en tant que tel pensant et parlant, sans avoir à se déguiser symboliquement en prince ou en oiseau pour habiter une signification et dire le monde.

\section{Ouvrir le corps de la sculpture: Anthony Caro}

Cette expérience du corps comme mise en présence du monde, de l'ouvert et du décentrement, il y a, à notre époque, une œuvre qui l'incarne avec une grâce particulière, en plus de son génie, de son audacieuse liberté et de sa fabuleuse inventivité - autant de choses qui nous rendent cette œuvre si aimable et si nécessaire pour vivre -, il s'agit de la sculpture d'Anthony

26. C. Greenberg, "Abstract and Representational», in The Collected Essays and Criticism, J. O'Brian (éd.), Chicago, The University of Chicago Press, vol. 3, Affirmations and Refusals, 1950-1956, 1993, p. 191.

27. M. Cunningham, Le danseur et la danse, entretiens avec J. Lesschaeve (éd. et trad.), Paris, Belfond, 198o, p. 77-78. 
Caro, et au premier chef (mais pas uniquement) des œuvres réalisées au cours des années 1960. Tout est geste dans cette sculpture, non pas au sens où le geste serait une expression (faire un signe à quelqu'un, mimer quelque chose), mais dans la mesure où le geste, dit Heidegger dans les Séminaires de Zurich, désigne "toute manière de se tenir, tout comportement où l'être humain se comporte comme un être-au-monde déterminé par l'être du corps, par la manière dont le corps donne corps ${ }^{28}$. Le geste, qui, en français comme en allemand, est issu de l'idée de porter (latin gero, ere: porter sur soi), caractérise la manière dont l'être humain porte l'existence en tant qu'il est un corps. Porter un enfant - la gestation - est à cet égard le geste des gestes; être à l'écoute d'un concert est un geste en ceci que par notre présence en corps, nous laissons le monde entrer en présence, le monde en tant que spatialité musicale, ce qui ne signifie pas un ensemble de distances mesurables, mais une dimension d'avoir-lieu, c'est-à-dire de tonalité vibratoire et de manifestation. (Pour en faire l'expérience en musique, par exemple, il suffit d'observer, comme y insiste le chef d'orchestre et phénoménologue Sergiu Celibidache, la différence entre le surgissement de la spatialité du monde devenu musique auquel nous assistons lors d'un concert où des musiciens, des instruments et nous-mêmes sommes présents en corps, et cette chose étrange, presque dépourvue de spatialité, que devient la musique quand nous l'écoutons en disque.)

Ainsi, tout geste est une manière d'exister le monde en corps et c'est en ce sens que la sculpture de Caro est geste - geste, plutôt que "gestuelle», afin d'éviter un contresens et une idée que Caro refuse absolument, comme il l'écrit dans une lettre du 15 juillet 1963 à son ami Michael Fried, celle du geste en tant qu' "action faite par le sculpteur». Rien n'est moins expressionniste que cette sculpture, qui est geste en ceci qu'elle nous ouvre au monde en tant que corps, en tant que nous nous tenons et existons debout sur le sol. Mais le geste n'est pas la traduction physique d'un contenu psychique intérieur, et la sculpture n'imite pas les mouvements d'un prétendu corps physique qui serait joint à une âme; affranchie de ce corps physique produit par la métaphysique occidentale, la sculpture de Caro se fait poème du corps, poème, plus précisément, de ce que le lecteur de Merleau-Ponty qu'est Michael Fried appelle " the livedness of the body» 29 : le corps tel qu'il est vécu et habité. En allemand, avons-nous déjà dit, le corps proprement humain, der Leib, est apparenté au verbe leben, vivre; c'est le corps qui donne vie au monde et qui se déploie dans une spatialité où n'est plus opérante la

28. M. Heidegger, GA 89, 787; Séminaires de Zurich, p. 145.

29. M. Fried, Anthony Caro [catalogue d'exposition, Hayward Gallery], Londres, Arts Council of Great Britain, 1969, p. 5. 
distinction entre l'intérieur et l'extérieur. Caro le dit à propos de son œuvre The Window (1966-1967): "Outside and inside are interrelated $»^{30}$. Ainsi, plus on marche autour de cette sculpture, moins on sait où se trouvent l'intérieur et l'extérieur, qui sont en permutation constante, grâce à la légèreté d'une danse, toute en acier peint, de panneaux, de grilles, de tubes de poutrelles: c'est un ballet de forces mises en tension (horizontales, verticales, obliques, vide, plein, transparence, sans parler du jeu de la lumière et de la poésie des contacts - tout un art du toucher - entre les éléments ou avec le sol), suivant un rééquilibrage qui, dans une poétique de purs rapports, se fait, se défait et se refait, incessamment et à chaque fois que se modifie l'angle de vue. Ni action ni expression (on entre avec Caro dans un espace sculptural purement abstrait, mais on y entre littéralement et de la manière la plus incarnée qui soit), la sculpture de Caro est geste en tant qu'ouverture radicale de notre corps au monde. L'ouvert, comme dit aussi bien Heidegger que Charles Olson, pourrait être un des qualificatifs principaux de l'œuvre de ce sculpteur qui a définitivement ouvert le corps de la sculpture (le bloc monolithique vertical) et déclôturé son aire de rayonnement en abolissant le traditionnel socle, de sorte que ses œuvres ne se présentent plus comme des objets d'exposition à regarder à partir de points fixes de visée. Comprenons bien : la suppression du socle ou du piédestal n'est pas chez Caro une nouvelle convention, mais à la fois la cause et le résultat d'une transformation complète du sens du sol, du déploiement de la sculpture et du rapport sol-sculpture. Comme le montrent de manière radicale Slow Movement (1965) ou Smoulder (1965), et de manière générale les œuvres réalisées pendant les années d'enseignement à Bennington College (1963-1965), le sol n'est plus un support pour quoi que ce soit, ni une donnée physique originaire (l'horizontal); il fait partie intégrante de la sculpture qui le doue de vie par un jeu de lignes, d'angles, de contacts et de mouvements sans cesse vivants et variables. Ainsi, de plain-pied avec notre corps entier, les œuvres de Caro libèrent un espace d'expérience de l'êtreau-monde, un espace en corps à directions multiples qu'aucun centre ne vient unifier, même si chaque sculpture constitue avec une précision extrême un tout qui nous comprend et nous ménage ainsi un horizon nécessaire à toute habitation humaine. "It has to do with presence, dit Caro dans un entretien avec Phyllis Tuchman, more as one person relates to another ${ }^{31}$, et nous pouvons penser aussi à la présence nue, ni conceptuelle (libre de tout discours théorique) ni figurative, des mégalithes qu'il vit à Carnac en 1959.

30. Caro by Anthony Caro, A. Renshaw (éd.), Londres, Phaidon Press, 2014, p. 146.

31. Cf. R. Whelan, Anthony Caro, Londres, Penguin Books, 1974, p. 120. 
Que veut dire ici présence? Une personne n'est ni un objet, ni quelque chose qui se tient en dehors ou à l'intérieur de nous. Être en présence de quelqu'un c'est partager l'ouverture même du monde, à travers une parole ou un silence mutuel, une pensée mutuelle, un geste mutuel. Être en présence d'une sculpture de Caro, c'est partager l'ouverture de la spatialité comme mise en présence du monde. Rien ne me semble aujourd'hui plus nécessaire que ces œuvres pour faire l'épreuve de ce que signifie être au monde en corps et mesurer à quoi nous engage la responsabilité phénoménologique fondamentale d'avoir à donner corps au monde, surtout en notre époque de gestion biologisante du corps et de " "biopolitique" de l'espèce humaine »" comme dit Michel Foucault dans les pages importantes de son cours de 1976.

Dans Contre la théâtralité (théâtralité et théâtre étant ici à comprendre comme mode de mise en scène de la subjectivité), Michael Fried souligne à juste titre les affinités entre cette œuvre et la modernité philosophique:

Les découvertes que fait Caro, à la faveur de son entreprise, sont aussi soudaines et impératives que n'importe quelle trouvaille de la philosophie moderne. [...] Tout se passe, avec Caro, comme si la sculpture avait été commise à une entreprise cognitive d'un nouveau genre: non que l'élan qui lui donne naissance soit devenu philosophique, mais il y a aujourd'hui un tel besoin explicite de vaincre le théâtre dans toutes ses manifestations que le projet d'interroger les divers modes d'être au monde pour faire de la sculpture n'est réalisable qu'à la condition de donner à cette interrogation une expression antilittérale, c'est-à-dire radicalement abstraite. (Au risque de paraître forcer l'argument, j'ajouterai que l'entreprise cognitive dont il s'agit a partie liée aussi bien avec la phénoménologie européenne qu'avec la philosophie du dernier Wittgenstein - seules les modalités diffèrent. [... $]^{33}$

Outre le beau texte de Merleau-Ponty «Le langage indirect et les voix du silence» dans Signes, auquel pense notamment Fried ${ }^{34}$, les deux textes de Heidegger Remarques sur art - sculpture - espace et L'art et l'espace

32. M. Foucault, Il faut défendre la société. Cours au Collège de France, 1975-1976, Paris, Gallimard - Seuil (Hautes études), 1997, p. 216 sq.

33. M. Fried, Contre la théâtralité. Du minimalisme à la photographie contemporaine, F. DurandBogaert (trad.), Paris, Gallimard, 1998, p. 105.

34. Dans un texte de 1993 où il revient sur l'événement que fut en 1960 la découverte de Midday de Caro et sur ses propres travaux consacrés au sculpteur, Fried écrit: «It was largely Midday too that I had in mind when I spoke in the Whitechapel introduction of the gestural and bodily aspect of Caro's art (I had been reading Maurice Merleau-Ponty, a fine preparation for Caro), and just this past week, teaching Merleau-Ponty's "Indirect Language and the Voices of Silence", it occurred to me, not for the first time, that the tension in that essay between a Saussurean conception of language as difference (i. e. as "pure" relation) and a thematics of gesture and embodiment perfectly captures the difficulty of adequately theorizing Caro's breakthrough achievement" (Caro by Anthony Caro, p. 37). 
développent à leur façon cette «entreprise cognitive d'un nouveau genre ». Heidegger n'ignore pas non plus les possibles de l'abstraction. Dans la lettre qu'il adresse à Bernhard Heiliger - sculpteur en partie abstrait -, il écrit: "Ihre Werke stellen nichts mehr dar - sie stellen uns in den Aufenthalt im Zwischen von Erde und Himmel - [Vos œuvres n'exposent plus rien - elles nous posent dans le séjour qui se tient dans l'entre-deux de la terre et du ciel - ]»35.

\section{L'épreuve du vide: Caro et Heidegger}

En quel sens - c'est la question que je voudrais poser pour finir - peut-on dire que l'" entreprise cognitive» de Caro (la manière dont cette ouvre découvre le monde et nous le fait connaitre) a une dimension essentiellement phénoménologique dont les résonances avec l'approche heideggerienne de la question sont manifestes, sans que Caro - ainsi que son fils Paul Caro a eu la grande amabilité de nous le confirmer - ait rien lu ni de Heidegger ni de Merleau-Ponty?

«Pour la première fois peut-être dans la culture occidentale, Caro a rendu explicite la possibilité de parler de l'expérience d'habiter au sein d'un corps ${ }^{36}$, écrit le sculpteur britannique Antony Gormley.

Reprenons les choses du côté de Caro: à partir de 1960, à partir de Twenty-Four Hours qui est sa première sculpture non figurative construite par assemblage d'éléments, Caro se libère de l'élément primordial de la sculpture traditionnelle, à savoir un bloc monolithique, une certaine masse soumise à la verticalité ainsi qu'à la pesanteur propres à tout corps, et un certain volume à modeler. Pour ce faire, il commence par agencer des éléments disparates qu'il ramène sur un plan, essentiellement frontal (comme dans Twenty-Four Hours ou Second Sculpture), bien que ce plan ne soit pas une surface uniforme; en effet, il est rendu respirant grâce à

35. Le texte manuscrit de la lettre est reproduit dans le petit catalogue de l'exposition Bernhard Heiliger, Saint-Gall, galerie Im Erker, 1964, p. 18. Voici une traduction du texte complet: "Cher Bernhard Heiliger, / Le moment passé dans votre atelier - j'aime mieux dire: là où l'œuvre a lieu [Werk-Statt] - m'a ouvert les yeux sur ce que votre œuvre est aujourd'hui à même de dire à nos contemporains et à ceux qui viendront. Dire signifie: montrer. / Et vous montrez l'éclosion de la terre dans le ciel terrestre qui nous est encore voilé. Vos œuvres n'exposent plus rien - elles nous posent dans le séjour qui se tient dans l'entredeux de la terre et du ciel - c'est le mouvement lui-même - qui prend son essor dans la libération du libre - c'est précisément lui qui devient manifeste - une "transfiguration" (pas une idéalisation) de l'être - à partir d'une source abritée en retrait. / Le recueillement du secret habite dans votre atelier où l'œuvre a lieu. / Un salut amical/ Votre / Martin Heidegger".

36. Caro by Anthony Caro, p. 23. 
des variations dans l'inclinaison des éléments ou à des ouvertures et des vides ménagés par la disparité des éléments. Ce dernier point est essentiel: en ne procédant plus à partir du modelage d'un volume de pierre ou de bronze, mais par assemblage, la sculpture de Caro intègre le vide comme composante majeure et amplifie ainsi considérablement son espace de rayonnement. À partir de cette effraction première, de cet éclatement inaugural du corps de la sculpture comme masse et volume, Caro travaille de plus en plus dans l'horizontalité, en étirant parfois à l'extrême les éléments dans la latéralité, comme par exemple dans Sculpture Seven (1961). Cet étirement latéral affranchit définitivement son œuvre de la traditionnelle verticalité sculpturale et lui ouvre ainsi un espace dont il ne va cesser d'explorer les possibilités nouvelles, un espace pour ainsi dire antigravitationnel, comme le sont les poupées dans le texte de Kleist Sur le théâtre de marionnettes, animées dans leur danse par rien d'autre que l'harmonie (Ebenmaß: la juste répartition des proportions), la mobilité (Beweglichkeit: l'être en mouvement) et l'aisance (Leichtigkeit: la facilité et l'agilité de ce qui ne pèse pas). Les conséquences sont aussi multiples qu'énormes, signalons celles qui sont phénoménologiquement les plus importantes.

Parce que le point de départ de la sculpture n'est plus la verticalité d'une masse, l'œuvre n'est plus soumise dans son principe d'assemblage aux lois de la pesanteur, si bien que des éléments qui peuvent pourtant être d'un poids énorme se mettent à jouer librement ensemble sans la moindre astreinte d'ordre physique. La cohérence de ce libre jeu des éléments affranchis de leur masse est renforcée par l'usage que Caro fait de la couleur dans les années 1960, couleur qui n'est pas appliquée extérieurement, mais qui participe de la logique interne de la sculpture et qui contribue, comme dit Caro, à accentuer "the mood of the work» ${ }^{37}$, la tonalité de l'œuvre, laquelle tonalité est aussi, comme on l'a vu avec Heidegger, un mode essentiel de la spatialité (la joie, la tristesse, toutes les tonalités, sont une manière d'habiter l'espace en corps). Ainsi, dans Midday, d'un jaune inoubliable, et même dans Sculpture Seven, d'énormes poutres en double T entrent en contact les unes avec les autres avec une légèreté qui introduit un tact d'un genre entièrement nouveau dans l'histoire de la sculpture. S'agissant de ce tact, William Rubin qui a réalisé en 1975 la grande rétrospective Anthony Caro au Museum of Modern Art (MoMA) observe très justement que dans les œuvres de Caro, les éléments «tendent à se toucher plus qu'à se chevaucher ou à se soutenir les uns les autres - une relation qui s'exprime techniquement par le fait

37. Ibid., p. 50 . 
qu'ils sont souvent vissés ou boulonnés ensemble plutôt que soudés ${ }^{38}$. Mais en plus de ce tact, qui est un des ressorts essentiels de la grâce de l'art de Caro, il faut comprendre que la libération, par l'horizontalité, de la physicalité de la masse, met en œuvre un bouleversement complet du principe de composition sculptural et, par voie de conséquence, du concept même d'espace.

En effet, l'œuvre n'étant pas élaborée dans la verticalité, n'étant donc plus soumise à la pesanteur, Caro n'est pas contraint de concevoir à l'avance comment il va faire tenir ensemble les éléments. Aucun dessin préalable n'est désormais nécessaire, si bien que la sculpture s'invente au fur et à mesure, dans la temporalité nouvelle d'une improvisation où la seule loi est celle de la cohérence interne des éléments entre eux. Il en résulte un espace purement sculptural qui émane de l'œuvre elle-même et dans lequel nous pouvons d'autant mieux entrer que la sculpture fait corps avec le sol sur lequel nous nous tenons également. Si nous n'entrons pas dans cet espace qu'aménage la sculpture, si nous la percevons comme un objet extérieur, l'œuvre ne peut apparaitre que comme un amas arbitraire de ferraille peinte ou rouillée. C'est pourquoi ces œuvres ne demandent pas que nous les contemplions et rompent ainsi avec le primat d'une certaine conception métaphysique et théorique du regard. Elles exigent en revanche que nous entrions avec elles dans une proximité, que nous nous tenions dans l'espace de leur présence, de leur phénoménalité sculpturale - ce qui ne veut pas dire pour autant pénétrer physiquement dans la sculpture, puisque c'est précisément parce que ces sculptures n'ont ni centre ni foyer intérieur qu'elles nous ménagent un espace qui est l'ouverture même. Seule une vraie expérience, un corps à corps, rend perceptible la réalité de cette ouverture de l'espace en monde que permet la sculpture. Mais une chose est sûre: il n'est pas possible de regarder statiquement une sculpture de Caro à partir d'un point fixe; quand bien même on le voudrait, l'œuvre insensiblement nous met en mouvement. On la regarde, ici, puis là, tout semble partir de l'œil, mais on tourne la tête, on se baisse, on se penche, peu à peu le corps tout entier est $\mathrm{mu}$, on avance, on recule, on se déplace latéralement, dans un sens, dans un autre, puis un autre encore, et comme la sculpture n'a pas d'orientation, pas de centre, pas de direction établie, elle nous désoriente dans nos gestes et nos habitudes corporelles; on fait alors quelques pas, on se met à marcher, lentement, longtemps, et en tous sens - après un certain temps, sans même s'en rendre compte, finalement: on danse. Mais

38. W. Rubin, Anthony Caro, Londres, Thames and Hudson, 1975, p. 50 (il s'agit d'une réédition du texte rédigé par Rubin pour le catalogue de l'exposition Anthony Caro, New York, MoMA, 1975). 
danser ne veut pas simplement dire qu'on bouge (il y a du reste de grands moments de danse dans l'immobilité). Danser signifie surtout être dépris de toute mécanicité - être libre -, du fait qu'on entre dans un rapport à soi et à l'espace tellement délié que le monde trouve sa juste amplitude et ainsi son sens. Justesse de placement, justesse du rythme, justesse du mouvement: la danse que libère la sculpture de Caro nous appelle pour donner corps au monde, c'est-à-dire soutenir le possible d'un juste et libre ordonnancement de ce qui est. Nous voilà ainsi vivant dans un espace de pure é-motion, l'espace de tous les gestes humains quand ils sont de vrais gestes, c'est-à-dire des gestes qui portent et font entrer en présence, pas des actions mécaniques: faire une caresse pour laisser entrer l'amour en présence, se tenir debout pour faire entrer la dignité en présence, offrir une main pour s'ouvrir à l'altérité (c'est aussi l'essence d'un poème pour Celan), regarder et écouter pour comprendre, tenir un violon pour donner corps à la musique, demeurer en silence pour faire place au deuil.

L'expérience de la sculpture abstraite ne s'oppose pas à une épreuve très concrète d'incarnation, tout au contraire. Comme l'écrit Dominique Fourcade:

C'est même le propre du travail de cette abstraction que d'indiquer au spectateur la juste place de son corps (à savoir : à l'extérieur des relations internes qui font que la sculpture en question s'établit comme sculpture), de lui indiquer aussi, qui plus est, le juste sens de ce corps. La grandeur de Caro est d'avoir compris intuitivement que seule une syntaxe aussi anti-littérale que possible, une syntaxe drastiquement abstraite, pouvait, dans la modernité de nos temps, rendre compte de notre corps et du monde - de l'implication de notre corps, c'est-à-dire de notre âme, dans le monde ${ }^{39}$.

Il y a comme un paradoxe, écrit Michael Fried, parce qu'on «fait une expérience des sculptures abstraites de Caro comme profondément physiques, mais pas, ou pas essentiellement, comme objets physiques ${ }^{40}$. L'essentiel est de bien comprendre que ni l'espace de la sculpture ni celui auquel nous donnons corps ne se laissent reconduire à des déterminations d'ordre physique au sens strict du terme. Par leur assemblage en sculpture, les

39. D. Fourcade, Antony Caro [catalogue d'exposition], Paris, galerie Piltzer-Rheims, 1977, p. 12.

40. Cf. M. Fried, Anthony Caro [catalogue d'exposition, Hayward Gallery], p. 11: «It may seem contradictory to claim that Caro's abstract sculptures are experienced as profoundly physical but not, or not essentially, as physical objects. The contradiction eases, however, and the continuity of his enterprise becomes fully clear, when it is recognized that this exactly characterizes the physicality of the body as actually lived. In Caro's art the physicality of the body is itself liberated from the body's limits». On a vu comment chez Heidegger aussi la question des limites du corps est phénoménologiquement déplacée. 
éléments matériels et physiques (une poutre d'acier, un tube d'aluminium) libèrent un espace d'un autre ordre, un espace de phénoménalité qui se déploie tout en gestes comme monde, avec ses lois propres - différentes de celle de la nature -, dont nous faisons l'épreuve de manière profondément incarnée. Dans les termes de Heidegger, cela signifie que l'espace physique - l'extension mesurable - qui est pensé depuis les Grecs à partir du corps physique [Körper] cède la place à ce qu'il nomme, en empruntant le mot à Goethe dans la Théorie des couleurs, l'espace comme "phénomène originaire [Urphänomen] ", c'est-à-dire l'espace comme dimension primordiale de monstration. Le point commun entre Caro et Heidegger - et il est à cet égard hautement significatif que Heidegger ait pensé ses textes en dialogue avec «l'art plastique moderne», comme il l'écrit dans L'art et l'espace ${ }^{41}-$, c'est d'abord qu'il s'agit chez l'un et chez l'autre d'une expérience de l'espace. C'est une évidence avec les œuvres de Caro, mais pour Heidegger aussi il est question de faire, à partir de la pensée, une expérience de l'espace. Qu'est-ce à dire? Que les deux courts textes de Heidegger ne sont pas une nouvelle théorie de l'espace comme le sont par exemple l'espace plan des parallèles régi par le cinquième postulat des Éléments d'Euclide ou l'espace en tant qu'extension tridimensionnelle uniforme qu'élabore mentalement Galilée. Lorsqu'il esquisse la loi d'inertie, dans la quatrième journée des Discours et démonstrations mathématiques concernant deux sciences nouvelles, Galilée écrit: "J'imagine [mente concipio] qu'un mobile a été lancé sur un plan horizontal d'où l'on a écarté tout obstacle ${ }^{42}$. Mente concipio, Heidegger analyse ce point en détail dans Qu'est-ce qu'une chose?, c'est une représentation de l'esprit, sans rapport direct avec l'espace tel que nous l'habitons.

Heidegger entreprend quant à lui de faire faire une expérience phénoménologique de l'espace, non pas de théoriser l'espace et de l'expliquer à partir de quelque chose d'autre que lui, en particulier le corps physique, comme c'est le cas dans la tradition philosophique depuis la Physique d'Aristote. Ainsi, son premier mouvement de pensée est d'affranchir l'espace de la corporéité physique pour éprouver l'espace en tant qu'espace, ou, écrit-il aussi: «faire l'expérience de ce que l'espace a de propre [das Eigentümliche des Raumes erfahren] " ${ }^{43}$. À partir de là, c'est le second mouvement, il est possible de voir comment il faut l'être humain pour que l'espace déploie sa spatialité, l'être humain en tant qu'il habite le monde et lui donne corps.

41. M. Heidegger, Die Kunst und der Raum/L'art et l'espace [éd. bilingue], J. Beaufret, F. Fédier (trad.), Saint-Gall, Erker-Verlag, 1969, p. 6 [all.] et p. 18 [fr.].

42. Galilée, Discours et démonstrations mathématiques concernant deux sciences nouvelles, M. Clavelin (éd. et trad.), Paris, PUF (Épiméthée), 1995, p. 205.

43. M. Heidegger, Die Kunst und der Raum/L'art et l'espace, p. 8 [all.] et p. 20 [fr.]. 
Comme le montre Proust au début de À la recherche du temps perdu: habiter une maison n'est pas occuper physiquement un certain volume, c'est, dans un rapport intime et tonal à nos gestes et à notre corps, libérer et faire place à tout un monde.

L'essentiel pour Heidegger est de penser l'espace non pas comme une chose ou un contenant, mais dans son déploiement propre: l'espace libère de l'espace, il «espace». Heidegger emploie ici le verbe räumen, directement parent du substantif der Raum, l'espace. Räumen, espacer, dit-il dans les Remarques sur art - sculpture - espace, «signifie essarter, libérer, dégager, donner libre champ à l'ouvert» ${ }^{44}$. Espacer, écrit-il encore dans L'art et l'espace, "cela fait advenir le libre, l'ouvert pour que puisse avoir lieu l'établissement et l'habiter des êtres humains ${ }^{45}$. En français, la manière à la fois la plus directe et la plus ouverte pour penser, comme Heidegger l'entend, le déploiement de l'espace, est peut-être la suivante: l'espace donne lieu. Ainsi, rien n'est moins uniforme que le propre de l'espace qui ne se déploie que si l'être humain le ménage et le laisse être grâce à sa manière de lui donner corps [Leiben], c'est-à-dire de déployer une habitation du monde. Il apparaît dès lors aussi que les choses ne sont en fait pas dans l'espace, mais que les choses sont en elles-mêmes des lieux [Orte]. Le lieu, quant à lui, n'est pas un contenant, une étendue géographiquement localisable. On parle de lieu ou de site quand s'ouvre une dimension d'entre-appartenance entre des choses; le lieu est ainsi la dimension inapparente pour que place soit faite à une rencontre. Rencontre il y a quand est ménagée une dimension essentielle d'encontre, que Heidegger nomme monde. Lieu et monde sont donc en très étroite affinité. Dans une maison qui nous est familière, les choses sont des lieux dont Marcel Proust s'emploie minutieusement à dire tout le sens tonal qui les habite pour former un monde. En Grèce, les temples sont aussi en eux-mêmes des lieux, des sites, parce qu'ils configurent un monde, ménagent l'espace où se rencontrent les dieux et les mortels, en articulant ensemble des éléments, en particulier, le rapport entre les colonnes et la lumière qui se déploie grâce au rythme de la taille de la colonne elle-même (sa cannelure) et au jeu des colonnes entre elles, lequel jeu est lui-même pris dans celui de l'alternance entre le plein des colonnes et le vide qui les unit. Mais pour chaque temple, chaque site, entre également en jeu l'articulation entre les éléments qui contribuent à faire le lieu: à Némée, l'articulation de la profondeur concave de la plaine et de la voussure du ciel, à Sounion entre la verticalité du roc, l'horizon de la mer qui s'étend tout autour et l'amplitude du ciel qui embrasse ciel et mer à la pointe extrême de ce cap.

44. M. Heidegger, Bemerkungen zu Kunst-Plastik - Raum, p. 13.

45. M. Heidegger, Die Kunst und der Raum/L'art et l'espace, p. 8 [all.] et p. 20 [fr.]. 
C'est au milieu des années 1930, dans L'origine de l'œuvre d'art, que Heidegger a formulé dans sa langue d'alors la manière dont l'espace architectural du temple grec est un lieu en tant qu'il institue un monde. Trente années plus tard, dans une langue en partie différente, sa formulation ultime de la pensée de l'espace l'amène à penser en dialogue avec la sculpture moderne, et à repenser ce qu'a de propre l'art plastique [das Eigentümliche der Plastik]. Pour bien saisir le déplacement qui se produit dans ces pages, au terme desquelles le vide n'est plus pensé comme un manque, comme le négatif du volume, mais comme un des modes d'institution des lieux, il faut savoir que plastique est issu du verbe grec plassô (ou plattô), qui veut dire modeler, façonner, donner forme en modelant une figure, un corps. On a vu que la sculpture de Caro, n'étant plus un art du modelé, n'est plus plastique en ce sens, avec toutes les conséquences que cela implique quant au sens de la sculpture et du rapport que nous entretenons avec elle. À la fin de L'art et l'espace, Heidegger tire de son côté les conclusions de sa pensée phénoménologique du déploiement de l'espace en tant qu'espace:

La plastique (la sculpture) serait la manière dont prennent corps des lieux, qui, en ouvrant une contrée et en la prenant en garde, tiennent rassemblés autour d'eux quelque chose de Libre, qui accorde aux choses de demeurer chaque fois en leur séjour et aux êtres humains d'habiter au beau milieu des choses.

S'il en est ainsi, que devient le volume des œuvres plastiques qui chaque fois font prendre corps à un lieu? Sans doute ne va-t-il plus délimiter les uns par rapport aux autres des espaces dans lesquels des surfaces enveloppent un intérieur faisant apparaître en contrepartie un extérieur. Ce qui est nommé du nom de «volume» devrait perdre son nom - dont la signification n'est pas plus ancienne que la science technique de la nature des Temps nouveaux ${ }^{46}$.

On pourrait croire ces lignes écrites pour Table Piece LXIV - The Clock (fig. 1), qui constitue une des œuvres de Caro les plus vides et les moins volumétriques qui soient, une des plus libres et des plus mélodieusement ouvertes, une des sculptures, pour tout dire, les plus ouvertement sculpture de l'histoire de la sculpture occidentale. Bien que Heidegger n'en sût rien, elle a été achevée un an avant l'écriture de ces lignes.

L'espace de l'habitation moderne que libère l'art plastique moderne n'est pas celui - régi par la physique - de la masse et du volume en tant qu'espace occupé par un corps. Il n'est pas non plus celui où, comme dans le temple grec, plein et vide confèrent, dans leur alternance et leur complémentarité, son rythme à un certain monde. Cet espace a une tout autre abyssalité, qui appelle une responsabilité nouvelle. Ce sont en effet

46. M. Heidegger, Die Kunst und der Raum/L'art et l'espace, p. 11-12 [all.] et p. 23-24 [fr.]. 


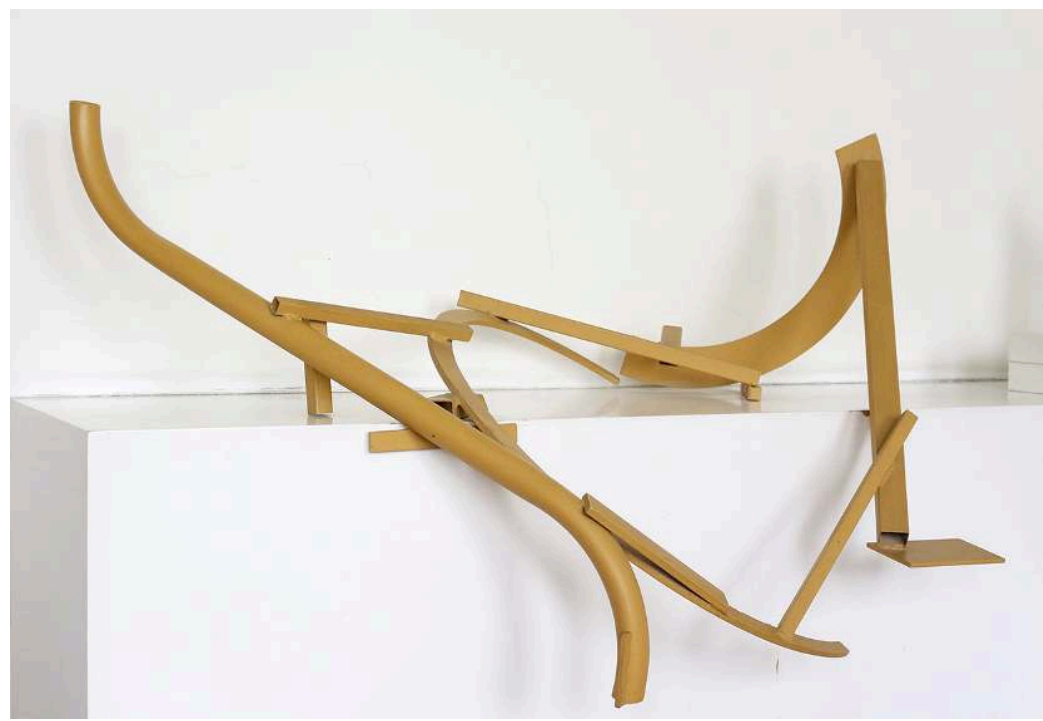

Fig. 1: Anthony Caro, Table Piece LXIV - The Clock (1968) - Steel, painted yellow, $30 \times 51 \times 32^{\prime \prime} / 76,2 \times 129,5 \times 81,3 \mathrm{~cm}$, (C) Courtesy of Barford Sculptures Ltd (cliché H. France-Lanord)

les distinctions mêmes du vide et du plein, de l'intérieur et de l'extérieur, qui ont perdu leur sens et qui nous engagent à découvrir une spatialité autre pour habiter le monde en corps. Cette spatialité, qui n'est plus celle du volume (ni le volume d'une caryatide de l'Érechthéion, ni l'espace pictural essentiellement volumétrique de l'homme de la Renaissance, ni le volume comme puissance centralisatrice du corps physique de l'hommesujet), interroge fondamentalement la place de l'être humain, ou plus exactement la manière pour l'être humain d'être à sa place et de donner corps. Libéré du volume, l'être humain se découvre livré à l'espace en tant qu'espace, dont il porte en corps la responsabilité sans en être pour autant le centre. Il apprend que être humain n'est pas le substantif d'une substance fondée ontologiquement en elle-même et encapsulée dans «le "boîtier" de la conscience» (Être et temps); c'est endurer la responsabilité d'un verbe: être, c'est-à-dire avoir à être ouvert, n'être que pur rapport, être au monde. Il résulte notamment de cette spatialité sans volume de l'être ouvert que l'humanité de l'homme moderne ne trouve plus en rien son fondement, mais a, en tant qu'être au monde, à répondre d'une justesse de placement par rapport au monde, qui implique à chaque fois les autres, les choses et soi. C'est aussi là que s'enracine la liberté qui est au cœur de la pensée 
heideggerienne de l'espace. Toutefois, Heidegger parle plus volontiers du libre, das Freie, que de «la liberté», puisque n'est pas en jeu la volonté d'un sujet, mais la dimension ouverte et désencombrée (du vouloir en particulier) au sein de laquelle l'être humain peut s'engager à laisser être.

Relisons ces lignes de L'art et l'espace: «Ce qui est nommé du nom de "volume" devrait perdre son nom [...]». Les implications de ce bouleversement sont, nous venons de le voir, considérables. Ce qu'énonce ici Heidegger peut également se dire de manière aussi diverse qu'inventive dans la langue de ses contemporains: Femme à la corbeille de Julio González (1934), Star Cage de David Smith (1950), El peine del viento I d'Eduardo Chillida (1954), Hop Scotch d'Anthony Caro, dont la justesse de placement est inoubliable.

Hadrien FranCE-LANORD 